Elsevier Science Publishers B.V., Amsterdam

\title{
Stability of coextrusion through converging dies
}

\author{
Nitin R. Anturkar *, Tasos C. Papanastasiou and James O. Wilkes \\ Department of Chemical Engineering. The University of Michigan, Ann Arbor. \\ MI 48109-2136 (U.S.A.)
}

(Received January 29, 1991; in revised form May 6, 1991)

\section{Abstract}

A linear stability analysis of multilayer flow of viscoelastic liquids through long, converging dies is performed by a rigorous two-dimensional analysis (2-D), as well as by a simplified one-dimensional analysis (1-D) on selected cross-sections along the die. The rheological behavior of liquids is represented by a modified Oldroyd-B model with a shear-rate dependent viscosity, described by the Carreau viscosity function. In the 2-D analysis, a streamlined Galerkin/finite-element method is used to discretize the domain. The resulting asymmetric generalized eigenvalue problem is large (of the order of 3000-8000), sparse, and banded, with a singular mass matrix. The leading eigenvalues of this complex problem are computed by using an iterative Arnoldi's algorithm, modified by Schur-Weilandt deflation, complex shift, and exponential preconditioning. With these series of modifications, the algorithm is now sufficiently flexible to solve any application that belongs to a generic class of large hydrodynamic stability problems.

The effect of the die geometry on the neutral stability curves is investigated for various operating conditions and rheological parameters. In all the investigated cases, the critical flow-rate ratios in long converging channels are found to be independent of the shapes, and of the ratios of the thicknesses at the inlet to the outlet of the die. These results agree well with the approximate, simplified 1-D analysis, indicating that the most dangerous instability is at the inlet of the die. Thus, the analysis of the entire two-dimensional flow domain is unnecessary, at least for long channels, except for validating the 1-D analysis. The results also indicate that mesh-in-

\footnotetext{
* Author for correspondence. Current address: Scientific Research Laboratory, Ford Motor Company, Mail Drop 3198, Dearborn, MI 48121-2053 (U.S.A.)
} 
dependent eigensolutions cannot be obtained using the 2-D analysis when leading eigensolutions exist at large wavenumbers.

Keywords: coextrusion; converging dies; multi-layer flow; Oldroyd-B model; viscoelastic liquids

\section{Introduction}

A wide range of desired properties can be achieved in plastic films by constructing a layerwise composite structure of polymers, in which each of these layers provides a specific end-use property, such as oxygen and moisture barrier, and thermoformability. These multilayer films are manufactured by coextrusion through converging dies, and are used in applications ranging from food-packaging to paper-lamination. At certain operating conditions, interfacial instability in the form of wavy interfaces is observed, which is detrimental to the product quality (see Fig. 1).

Interfacial instability in multilayer plane Poiseuille flow of Newtonian liquids was investigated using a linear stability analysis [1-4]. The researchers found that the onset of instability occurs even at vanishingly small Reynolds number in the presence of viscosity stratification. A similar analysis of multilayer extrusion of viscoelastic liquids indicated, however, that elasticity provides a stabilizing effect, such that operating windows of

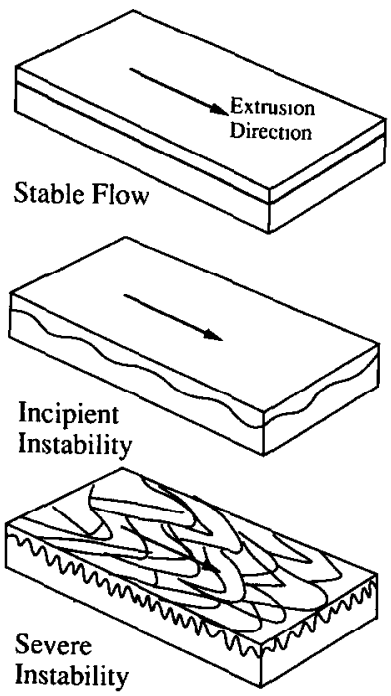

Fig. 1. Interfacial instability in multilayer extrusion. 
stable conditions at all wavelengths could be constructed [5-7]. Khomami [8] investigated interface deformation when two immiscible liquids merge together in a converging channel, in which he predicted that the normal stresses play a major role in interfacial stability. A limited number of experimental studies were also performed $[9,10]$. However, they were inadequate to establish any general criteria of interfacial instability. The experiments by Han and Shetty [9] had insufficient rheological data, whereas Kao and Park [10] investigated shear-mode instability rather than interfacial-mode instability.

A typical industrial die has dimensions of $3-5 \mathrm{~cm}$ length, $30-40 \mathrm{~cm}$ width and $0.2-0.3 \mathrm{~cm}$ depth at the inlet [11], and has a converging shape from the inlet to the outlet. The effect of this shape on the stability of multilayer flow has not been addressed in the literature, even for Newtonian liquids. In this paper, we will investigate the effects of the shape of converging dies, and the ratios of their depth at the inlet to the outlet on the stability of multilayer flows of viscoelastic liquids.

Since typical industrial dies are by an order-of-magnitude longer than their depth, two approaches are possible. In one approach, the steady-state flow is a two-dimensional converging flow (2-D) with the variation in velocity along the depth and the length, whereas in another approach, the flow is assumed to be one-dimensional channel flow locally at various cross-sections of the die (1-D). Two-dimensional linear stability analyses are

\section{TABLE 1}

Differences between the numerical schemes in the 1-D and the 2-D approaches

1-D approach

(1) 1-D steady-state flow locally at various cross-sections

(2) Applicable only to long dies

(3) Pseudospectral discretization

(4) Shooting technique for predicting eigenvalues

(5) Disturbance:

$\bar{f}(x, y, t)=\mathrm{f}^{*}(y) \exp (\mathrm{i} \alpha x) \exp (c t)$

(6) A standard numerical technique
2-D approach

2-D steady-state flow

Applicable to any die

Streamlined Galerkin/finite-element method

A modified Arnoldi's algorithm for predicting eigenvalues

Disturbance:

$\bar{f}(x, y, t)=\mathrm{f}^{*}(x, y) \exp (c t)$

A novel numerical technique with a wide range of applications.

The variable $f$ represents the flow variables, $\alpha$ the real wavenumber, $c$ the complex wavespeed, $x$ the direction along the flow, $y$ the direction along the depth of the die, and $t$ the time. 
carried out for both approaches. However, a convenient Fourier normalmode decomposition of disturbances along the direction of flow can only be incorporated in the 1-D analysis. The linear stability analysis in the 1-D approach, which is similar to the analysis of the channel flow reported by Anturkar et al. [6,7], is performed at various cross-sections of the die to determine the most unstable mode. In this paper, only the 2-D approach is described in detail. However, the essential differences between the numerical schemes of the two approaches are summarized in Table 1 . Note that the 2-D analysis is computationally expensive. Besides, for the case in which the leading eigenvalues exist at large wavenumbers, the results are mesh-dependent (see Section 5). In these cases, the 2-D analysis is ineffective.

The steady-state-analysis in the 2-D approach, carried out by means of the streamlined Galerkin/finite-element method with Newton's iteration is described in Section 2. The formulation of the linear stability analysis with finite elements is described in Section 3. The discretization of the two-dimensional flow domain gives rise to a large, asymmetric generalized eigenvalue problem. Computations of the leading eigenvalues of such a complex problem using a modified Arnoldi's algorithm are discussed in Section 4. The results obtained from both the approaches are presented and compared in Section 5, and the conclusions are summarized in Section 6.

\section{Steady-state analysis}

A schematic flow geometry and the coordinates of the two-dimensional flow in a converging channel are shown in Fig. 2. Although a two-layer flow is shown here, the algorithm is developed for l-layer flow, where the number of layers $l$ is arbitrary. The dimensionless equations of change are Continuity equation:

$\nabla \cdot v_{j}=0, \quad j=1,2, \ldots, l$.

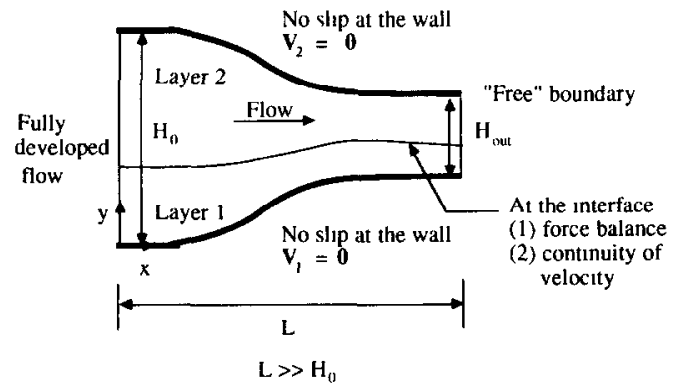

Fig. 2. A schematic diagram of the flow geometry, coordinate axes and boundary conditions. 
Conservation of momentum equation:

$R e_{J}\left[\frac{\partial v_{j}}{\partial t}+v_{j} \cdot \nabla v_{j}\right]=-p_{J} \nabla \cdot I+\nabla \cdot \tau_{j} \quad j=1,2, \ldots, l$.

where, the subscript $j$ denotes the $j$ th layer. The variable $\tau_{j}$ is the stress tensor, $v_{j}$ the velocity vector, $p_{j}$ the pressure, $R e$, the Reynolds number, and $I$ is the unit tensor. The equations are made dimensionless by the total average velocity $V$, the total thickness $H_{0}$ of the die at the inlet, and by the zero shear-rate viscosity $\eta_{01}$ of the first layer. Henceforth, all equations are for $j=1,2, \ldots, l$, unless otherwise specified.

A modified Oldroyd-B model with shear-dependent viscosity is chosen as a constitutive equation to relate the stress tensor $\tau_{j}$ to the rate-of-strain tensor $\dot{\gamma}_{j}$. The viscosity $\boldsymbol{\eta}$ is a function of the magnitude $\dot{\gamma}\left(=\left[\frac{1}{2} \dot{\gamma}: \dot{\gamma}\right]^{1 / 2}\right)$ of the rate-of-strain tensor, and is given by the Carreau viscosity function [12]. The constitutive equation in its dimensionless form is

$\tau_{\jmath}+\Lambda_{1 \jmath} \tau_{(1) \jmath}=\Gamma_{J}\left(\dot{\gamma}_{J}\right)\left[\dot{\gamma}_{J}+\Lambda_{2 j} \dot{\gamma}_{(2) \jmath}\right]$,

where $\Gamma_{j}\left(\dot{\gamma}_{j}\right)=\kappa_{j}\left[1+\Lambda_{v j}^{2} \dot{\gamma}_{\jmath}^{2}\right]^{(n,-1) / 2}$ is the dimensionless Carreau viscosity function, $\kappa_{J}=\eta_{0 j} / \eta_{01}$ is the zero-shear-rate viscosity ratio, $n$, the power-law exponent, and $\Lambda_{v j}$ is the time constant in the viscosity function. The dimensionless time constants $\Lambda_{1}$, and $\Lambda_{2}$, in the Oldroyd-B model are the relaxation and retardation times respectively. The convected derivatives of the stress tensor $\tau_{(1) \jmath}$, and the rate-of-deformation tensor $\dot{\gamma}_{(2) J}$ are

$$
\begin{aligned}
& \tau_{(1) \jmath}=\frac{\partial \tau_{j}}{\partial t}+v_{j} \cdot \nabla \tau_{j}-\left[\tau_{j} \cdot \nabla v_{j}+\left(\nabla v_{j}\right)^{\dagger} \cdot \tau_{j}\right], \\
& \dot{\gamma}_{(2) J}=\frac{\partial \dot{\gamma}_{J}}{\partial t}+v_{j} \cdot \nabla \dot{\gamma}_{J}-\left[\dot{\gamma}_{j} \cdot \nabla v_{j}+\left(\nabla v_{j}\right)^{\dagger} \cdot \dot{\gamma}_{j}\right] .
\end{aligned}
$$

At low shear rate, the dimensionless viscosity $\Gamma_{J}\left(\dot{\gamma}_{j}\right)$ approaches $\kappa_{J}$, and at high shear rates, it exhibits power-law behavior. The power-law model predicts unrealistically high viscosity at low shear rates. Therefore, for pressure-driven flows involving regions of low shear rates, the Carreau viscosity function is an appropriate choice rather than the power-law model. In general, fluid particles experience extensional flow in the converging channel. The Oldroyd-B model is unsuitable for extensional flows, because of its unrealistic prediction of an infinite extensional viscosity at a finite extension rate [12]. However, shear flow dominates over extensional flow in long dies, and the extension rate never approaches the value at which the elongational viscosity is unrealistically high. 
The modified Oldroyd-B model can also be written as [13,14],

$$
\begin{aligned}
& \tau_{J}=\tau_{v j}+\tau_{e j}, \\
& \tau_{v j}+\Lambda_{1 j} \tau_{v(1) j}=\Gamma_{v j}\left(\dot{\gamma}_{J}\right) \dot{\gamma}_{j}, \\
& \tau_{e J}=\Gamma_{e j}\left(\dot{\gamma}_{J}\right) \dot{\gamma}_{J}, \\
& \Gamma_{J}\left(\dot{\gamma}_{J}\right)=\Gamma_{e j}\left(\dot{\gamma}_{j}\right)+\Gamma_{v j}\left(\dot{\gamma}_{j}\right), \\
& \Lambda_{2 J}=\Lambda_{1} \frac{\Gamma_{e j}\left(\dot{\gamma}_{j}\right)}{\Gamma_{j}\left(\dot{\gamma}_{j}\right)} .
\end{aligned}
$$

The above rearrangement of the constitutive equation can be considered to be a model of a viscoelastic liquid with relaxation time $\Lambda_{1 j}$ and viscosity function $\Gamma_{v j}\left(\dot{\gamma}_{j}\right)$ in a shear-thinning, inelastic solvent with viscosity function $\Gamma_{e j}\left(\dot{\gamma}_{j}\right)$. The subscripts $e$ and $v$ correspond to the inelastic and viscoelastic components respectively. It is assumed that $\Gamma_{j}\left(\dot{\gamma}_{j}\right), \Gamma_{e j}\left(\dot{\gamma}_{j}\right)$, and $\Gamma_{v j}\left(\dot{\gamma}_{j}\right)$ have the same functional form with the same time constant $\Lambda_{v j}$ and the same power-law exponent $n_{j}$ in the model. The only difference in these three viscosity functions is their values at zero shear rate. The retardation time $\Lambda_{2 j}$ is assumed to be equal to $\Lambda_{1 j} / 9$ in all the calculations. Equations (2), (3) and (6) are then rearranged such that,

Conservation of momentum equation:

$R e_{j}\left[\frac{\partial v_{j}}{\partial t}+v_{j} \cdot \nabla v_{j}\right]=\nabla \cdot\left(-p_{j} I+\tau_{v_{j}}+\frac{\Lambda_{2 j}}{\Lambda_{1 j}} \Gamma_{j}\left(\dot{\gamma}_{J}\right) \dot{\gamma}_{j}\right)$

Constitutive equation:

$\tau_{v J}+\Lambda_{1 J} \tau_{v(1) J}=\left(1-\frac{\Lambda_{2 J}}{\Lambda_{1 j}}\right) \Gamma_{J}\left(\dot{\gamma}_{J}\right) \dot{\gamma}_{J}$.

Since the relationship between $\Gamma_{j}\left(\dot{\gamma}_{j}\right), \Gamma_{v j}\left(\dot{\gamma}_{j}\right)$ and $\Gamma_{e j}\left(\dot{\gamma}_{j}\right)$ is known, the above formulation does not introduce any additional unknowns. Henceforth, the subscript $v$ is removed from $\tau_{v j}$ for simplicity. This arrangement of the governing equations is preferred for numerical stability in Galerkin/ finite-element analysis, and also for a better physical interpretation of the constitutive equation.

The velocities, stresses, pressure, and interfacial locations are determined for steady-state flow by solving eqns. (1), (7) and (8) in the absence of time-derivatives. The boundary conditions in terms of dimensional variables are (see Fig. 2):

(1) no slip at the stationary walls: $\boldsymbol{v}_{\text {wall }}=0$;

(2) continuity of velocity at interfaces: $v_{j}=v_{j+1}$;

(3) force balance at interfaces: $\boldsymbol{n}_{j} \cdot\left(\tau_{j}-\tau_{j+1}+p_{j+1} \boldsymbol{I}-p_{j} \boldsymbol{I}\right)+\boldsymbol{n}_{j} \cdot \tilde{\boldsymbol{H}}_{j} /$ $\mathrm{Ca}=0$; 
(4) kinematic boundary condition: $n_{j} \cdot\left(v_{j}-\partial h_{j} / \partial t\right)=0$, where, $h_{j}$ is the distance of the $j$ th interface from the base level, $\boldsymbol{n}_{j}$ the unit normal vector and $\tilde{\boldsymbol{H}}_{j}$ the curvature vector, and the capillary number $\mathrm{Ca}$ is defined as $V \eta_{01} / \sigma_{j}$, where $\sigma_{j}$ is the interfacial tension between the $j$ th and the $(j+1)$ th layers.

The assumption of fully developed flow at the inlet of the die, which is a valid assumption for the multilayer extrusion process, specifies the boundary conditions on stresses and velocities at the inlet of the die. These boundary conditions are computed by solving the equations for one-dimensional multilayer plane Poiseuille flow [6,7]. Since the flow rates are easily measurable quantities compared to the thicknesses of the individual layers, they are computed along with velocities and stresses for a given set of thicknesses of all layers, and the results are reported in terms of the flow rates. At the outlet of the die, a weak form of the governing equations in finite-element analysis is extended to the outflow boundary. This "free" boundary condition has been proposed by Papanastasiou et al. [15], and has been tested for several applications by Malamataris et al. [16].

The streamlined Galerkin/finite-element method is used to solve the steady-state equations, in which the elements align themselves along streamlines [17]. The locations of the nodes of the elements are unknown variables,

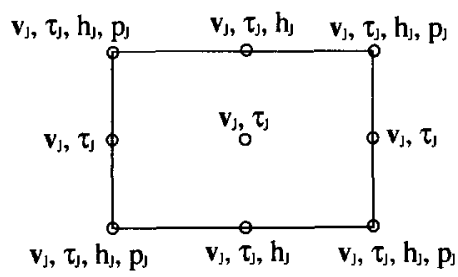

(a) Element is not on the interface

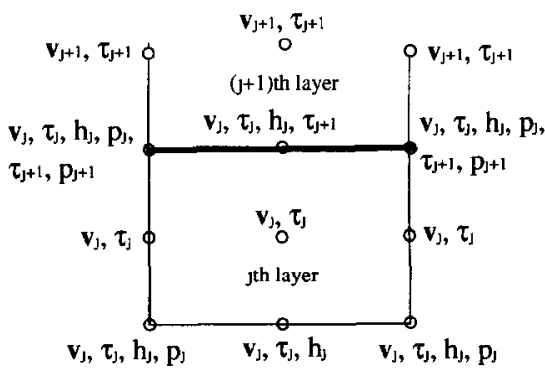

(b) Element is on the interface

Fig. 3. The nodal unknowns (a) for an element in the flow domain not on the interface, and (b) for an element just below the interface. 
which fall on the streamlines by satisfying the kinematic condition of no mass penetration. Since the interfaces are along the streamlines, the interfacial locations are simultaneously determined along with the nodal pressures, velocities and stresses by solving the kinematic equation simultaneously with the equations of change. In our analysis, the $x$-coordinates of all the nodes are fixed, and the $y$-coordinates are left as unknowns. The formulation of the Galerkin / finite-element method is customary, and is described elsewhere [18]. With this formulation, the degrees of freedom in a typical element away from the interface, and in one adjacent to the interface are shown in Fig. 3. Note that there are two unknown stress tensors and two unknown pressures per interfacial node, corresponding to two adjacent layers.

The resulting nonlinear algebraic equations are solved by Newton's iteration

$\boldsymbol{J}\left(\boldsymbol{x}^{k}\right)\left[\boldsymbol{x}^{k+1}-\boldsymbol{x}^{k}\right]=-\boldsymbol{R}\left(\boldsymbol{x}^{k}\right)$,

where $\boldsymbol{J}=\partial \boldsymbol{R} / \partial \boldsymbol{x}$ is the Jacobian matrix, $\boldsymbol{x}$ the vector of the unknowns $\boldsymbol{v}_{f}$, $\tau_{j}, p_{j}$ and $h_{f}$, and the superscript $k$ represents the values evaluated at the $k$ th iteration. The set of linear equations in eqn. (9) is then solved by the frontal routine developed by Hood $[19,20]$ for asymmetric matrices.

\section{Linear stability analysis}

The methodology of the linear stability analysis is standard, and is described in several books (e.g. Drazin and Reid [21]). However, implementation of the stability analysis of the two-dimensional steady-state flow is rarely reported in the literature. In these flows, conventional Fourier normal-mode decomposition of disturbances cannot be implemented, and the resulting linear equations are two-dimensional.

The superposition of a small perturbation on a steady base flow can be represented as

$f_{j}(x, y, t)=f_{s j}(x, y)+\hat{f}_{j}(x, y, t)$,

where the variable $f$ represents the velocities, stresses, and pressure, the subscript $s$ denotes the steady-state variable, and the overcap represents the perturbed variable. The locations of the streamlines, which are unknowns in the streamlined finite elements, are also perturbed and represented as

$h_{j}(x, t)=h_{s_{j}}(x)+\hat{h}_{j}(x, t)$.

Equations (10) and (11) are substituted in eqns. (1), (7) and (8), and only the 
linear terms in the disturbances are retained for infinitesimally small disturbances. The resulting equations are

$\nabla \cdot \hat{v}_{j}=0$

$R e_{j}\left[\frac{\partial \hat{\boldsymbol{v}}_{j}}{\partial t}+\boldsymbol{v}_{s j} \cdot \nabla \hat{\boldsymbol{v}}_{j}+\hat{\boldsymbol{v}}_{j} \cdot \nabla \boldsymbol{v}_{s j}\right]=\nabla \cdot\left[-\hat{p}_{j} \boldsymbol{I}+\hat{\boldsymbol{\tau}}_{j}+\frac{\Lambda_{2 j}}{\Lambda_{1 j}}\left(\Gamma_{s j} \hat{\dot{\gamma}}_{j}+\hat{\Gamma}_{j} \dot{\boldsymbol{\gamma}}_{s j}\right)\right]$

$\hat{\tau}_{j}+\Lambda_{1 j} \hat{\tau}_{(1) J}=\left(1-\frac{\Lambda_{2 j}}{\Lambda_{1 J}}\right)\left(\Gamma_{s j} \hat{\dot{\gamma}}_{j}+\hat{\Gamma}_{j} \dot{\gamma}_{s J}\right)$

where

$$
\begin{aligned}
\hat{\tau}_{(1) J} & =\frac{\partial \hat{\tau}_{j}}{\partial t}+v_{s J} \cdot \nabla \hat{\tau}_{j}+\hat{v}_{j} \cdot \nabla \tau_{s j} \\
& -\left[\tau_{s j} \cdot \nabla \hat{v}_{j}+\hat{\tau}_{j} \cdot \nabla v_{s J}+\left(\nabla v_{s j}\right)^{\dagger} \cdot \hat{\tau}_{j}+\left(\nabla \hat{v}_{j}\right)^{\dagger} \cdot \tau_{s j}\right], \\
\hat{\Gamma}_{J} & =\frac{(n,-1) \Lambda_{v j}^{2} \dot{\gamma}_{s J}}{1-\Lambda_{v j}^{2} \dot{\gamma}_{s J}^{2}} \Gamma_{s j} \hat{\dot{\gamma}}_{J} .
\end{aligned}
$$

The steady-state quantities in these equations have already been determined by the prior analysis in Section 2. Equations (10) and (11) are also substituted in the boundary conditions described in Section 2, and subsequently linearized.

Since the governing equations and the boundary conditions are linear in disturbances, the behavior of the disturbed variable with respect to time is exponential. Then the finite-element expansions for the disturbances $\hat{\boldsymbol{v}}_{J}, \hat{\boldsymbol{\tau}}_{f}$, $\hat{p}_{J}$ and $\hat{h}_{j}$ are

$$
\begin{aligned}
& \hat{\boldsymbol{v}}_{j}(x, y, t)=\sum_{k} \hat{\boldsymbol{v}}_{j k} \phi_{k}\left\{x_{p}, y_{p}\right\} \exp (c t), \\
& \hat{\tau}_{j}(x, y, t)=\sum_{k} \hat{\tau}_{j k} \phi_{k}\left\{x_{p}, y_{p}\right\} \exp (c t), \\
& \hat{p}_{j}(x, y, t)=\sum_{k} \hat{p}_{j k} \psi_{k}\left\{x_{p}, y_{p}\right\} \exp (c t), \\
& \hat{h}_{j}(x, t)=\sum_{k} \hat{h}_{j k} \phi_{k}\left\{x_{p}, y_{p}=1 \text { or }-1\right\} \exp (c t),
\end{aligned}
$$

where $c=c_{\mathrm{R}}+\mathrm{i} c_{\mathrm{I}}$ is the complex velocity of disturbances, and $\hat{\boldsymbol{v}}_{j k}, \hat{\tau}_{j k}, \hat{p}_{j k}$ and $\hat{h}_{j k}$ are the nodal coefficients of the amplitudes of the perturbations of the respective variables. When $c_{\mathrm{R}}>0$, the flow is temporally unstable; when $c_{\mathrm{R}}<0$ the flow is temporally stable; and when $c_{\mathrm{R}}=0$, the flow is neutrally stable. The imaginary part $c_{\mathrm{I}}$ is the temporal oscillation rate.

A correction is required in the acceleration terms due to the deformation of elements with time. These terms, such as $\partial \hat{v}_{J} /\left.\partial t\right|_{x, y}$ and $\partial \hat{\tau}_{j} /\left.\partial t\right|_{x, y}$, are 
with respect to the laboratory coordinates, and should be expressed in isoparametric coordinates used in the Galerkin/finite-element formulation. The correlation is,

$\left.\frac{\partial f}{\partial t}\right|_{x_{p}, y_{p}}=\left.\frac{\partial f}{\partial t}\right|_{x, y}+\frac{\mathrm{d} y}{\mathrm{~d} t} \frac{\partial f}{\partial y}$

where $\mathrm{d} y / \mathrm{d} t$ is the velocity of a node in the isoparametric coordinates. The variable $f$ represents the velocity vector in the momentum equation or the stress tensor in the constitutive equation. No correction in eqn. (18) is required for the acceleration term $\partial h_{J} / \partial t$ in the kinematic condition. Note that in our finite-element analysis, only the $y$-coordinate of the nodal location varies with time, and therefore, the velocity of the nodal point is only along the $y$-direction.

The nodal locations themselves are the superposition of the steady-state and the disturbed coordinates. Consequently, the gradients and the Jacobian of the isoparametric transformation depend on the perturbation of the nodal coordinates. Therefore, additional linearization of the residuals is necessary to obtain the equations that are linear not only in disturbed velocities, stresses and pressures, but are also linear in the disturbed nodal locations [22].

When Galerkin/finite-element residuals are defined and boundary conditions are imposed, the resulting set of equations forms an asymmetric generalized eigenproblem with the vector of unknown disturbance coefficients $\hat{\boldsymbol{x}}$ as an eigenfunction, and with the complex wave propagation speed $c$ as an eigenvalue. This eigenproblem is represented as

$\boldsymbol{J} \hat{\boldsymbol{x}}=c \boldsymbol{M} \hat{\boldsymbol{x}}$,

where $\boldsymbol{J}=\partial \boldsymbol{R} / \partial \hat{\boldsymbol{x}}$ is the Jacobian matrix, $\boldsymbol{M}=\partial \boldsymbol{R} /\left.(\partial \boldsymbol{x} / \partial t)\right|_{x_{p}, y_{p}}$ is the mass matrix, and

$$
\begin{gathered}
\hat{\boldsymbol{x}}=\left[\ldots, \hat{\boldsymbol{v}}_{j 1}, \hat{\boldsymbol{v}}_{j 2}, \ldots, \hat{\boldsymbol{v}}_{j k n}, \hat{\tau}_{j 1}, \hat{\boldsymbol{\tau}}_{j 2}, \ldots, \hat{\boldsymbol{\tau}}_{j k n}, \hat{\boldsymbol{p}}_{j 1}, \hat{\boldsymbol{p}}_{j 2}, \ldots, \hat{\boldsymbol{p}}_{j l n}, \hat{h}_{j 1}, \hat{h}_{j 2}, \ldots,\right. \\
\left.\hat{h}_{j m n}, \ldots\right]^{\dagger},
\end{gathered}
$$

where $k n$ is the number of velocity or stress nodes, $\ln$ the number of pressure nodes, and $m n$ is the number of unknown nodal locations. The Jacobian in eqn. (19) is identical to the Jacobian in eqn. (9). The only non-zero contributions in the mass matrix are due to acceleration terms, and hence it is singular.

\section{Numerical algorithm}

In hydrodynamic stability analysis, one is interested only in leading eigenvalues, i.e. the eigenvalues having the largest real parts. Besides, in the 
generalized eigenvalue problem under consideration, both $\boldsymbol{J}$ and $\boldsymbol{M}$ are asymmetric, large (typically of the order of 3000-8000), sparse and banded, and $\boldsymbol{M}$ is singular. These features rule out most of the widely used methods, such as the QR algorithm [23-25], that compute all the eigenvalues or require full matrix storage. Newton's method, which is much simpler to implement than other alternatives, is used in the 1-D approach [6,7]. For the unknown eigenvector and eigenvalue, eqn. (19) and an additional constraint of any convenient normalization of the eigenvector are required. However, complex arithmetic and a dependence of the converged solution on the initial estimate make it unsuitable for large matrices in eqn. (19).

There are three other basic algorithms for solving large asymmetric eigenproblems $\boldsymbol{A x}=\boldsymbol{c x}$. They are: (1) subspace iteration [25]; (2) Lanczos' algorithm for asymmetric matrices [26]; and (3) Arnoldi's algorithm [27]. All three methods are based on oblique or orthogonal projections of $\boldsymbol{A}$ onto subspaces, such that $\boldsymbol{A}$ reduces to matrices, for which eigenvalues can be easily and quickly computed. They utilize a capability of premultiplying a vector by $\boldsymbol{A}$, thereby conserving the banded structure. However, the most dominant eigenvalues (with the largest absolute value), which are often not the leading ones, are computed. Besides, if $\boldsymbol{M}$ is perturbed slightly so that it is no longer singular, then some very large eigenvalues appear that grow unboundedly as the perturbation is reduced to zero [28]. To suppress these artificial eigenvalues, and to map the dominant eigenvalues to the leading eigenvalues, a transformation of the domain is required. In subspace iteration, the convergence is slower than that in other methods, whereas in Lanczos' algorithm, two orthogonal subspaces are needed, which increase the computational cost. Therefore, we have chosen Arnoldi's algorithm in our investigation. It is modified by Saad [29] and Christodoulou and Scriven [30] so that it can be efficiently used for hydrodynamic stability analysis. The additional modifications are incorporated in this paper to make the algorithm suitable even for stiff systems.

In the original Arnoldi's algorithm, the orthogonal projection of $\boldsymbol{A}$ onto a subspace $\boldsymbol{Q}_{m}$, spanned by the Krylov vectors $\left\{\boldsymbol{q}_{1}, \boldsymbol{A} \boldsymbol{q}_{1}, \boldsymbol{A}^{2} \boldsymbol{q}_{1}, \ldots, \boldsymbol{A}^{m-1} \boldsymbol{q}_{1}\right\}$ approximates the eigenpair $\{c, \boldsymbol{x}\}$ of $A$ by a pair $\left\{c_{m}, \boldsymbol{x}_{m}\right\}$, such that $\boldsymbol{x}_{m}$ belongs to $\boldsymbol{Q}_{m}$, and the residual of $\left(\boldsymbol{A}-c_{m} \boldsymbol{I}\right) \boldsymbol{x}_{m}$ is orthogonal to each column of $\boldsymbol{Q}_{m}$, i.e.

$$
Q_{m}^{\dagger}\left(\boldsymbol{A}-c_{m} \boldsymbol{I}\right) \boldsymbol{x}_{m}=0,
$$

where $\boldsymbol{q}_{1}$ is an arbitrary unit vector. In practice, rather than building $\boldsymbol{Q}_{m}$ sequentially using Krylov vectors, the algorithm produces an orthogonal basis of $\boldsymbol{Q}_{m}$. The projection of $\boldsymbol{A}$ on $\boldsymbol{Q}_{m}$ in the new orthogonal basis is represented by an upper Hessenberg matrix $H_{m}=Q_{m}^{\dagger} A Q_{m}$. The procedure 
to build $\boldsymbol{H}_{m}$ is

$$
\begin{aligned}
& \text { 1. } \overline{\boldsymbol{q}}_{l+1}=\boldsymbol{A} \boldsymbol{q}_{l}-\sum_{k=1}^{l} h_{k l} \boldsymbol{q}_{k}, \quad l=1,2, \ldots, m, \text { with } h_{k l}=\boldsymbol{q}_{k}^{\dagger} \boldsymbol{A} \boldsymbol{q}_{l}, \\
& \text { 2. } h_{l+1, l}=\left[\overline{\boldsymbol{q}}_{l+1}^{\dagger} \cdot \overline{\boldsymbol{q}}_{l+1}\right]^{1 / 2}, \\
& \text { 3. } \boldsymbol{q}_{l+1}=\overline{\boldsymbol{q}}_{l+1} / h_{l+1, l},
\end{aligned}
$$

where $h_{k l}$ is the $(k, l)$ th element of $\boldsymbol{H}_{m}$. The eigenpairs of the much smaller system (of the order $m$ ) of the Hessenberg matrix $\boldsymbol{H}_{m}$ are solved routinely by using EISPACK software. The eigenpairs of $\boldsymbol{H}_{m}$ are the Galerkin approximations of the eigenpairs of $\boldsymbol{A}$. Several modifications are, however, required before the above algorithm can be used.

(1) Iterative procedure. The number of Arnoldi steps, $m$, required to ensure convergence of desired eigenvalues is equal to $N^{1 / 2}$ [31], where $N$ is the order of the system. Therefore, an iterative procedure is used for large systems, in which the process is restarted after every $m$ steps. The initial vector of each successive iteration is a linearized combination of the normalized approximate eigenvectors computed in the previous iteration.

(2) Gram-Schmidt reorthogonalization. Computation of $\boldsymbol{q}_{l+1}$ by the procedure described in eqn. (21) usually involves large errors due to the subtraction of large numbers of approximately equal magnitude. The resulting set of columns can be far from orthonormal. Saad [31] employed a modified Gram-Schmidt reorthogonalization process to correct the loss of orthogonality only when important cancellations occur, thereby saving considerable amounts of computation time.

(3) Schur-Weilandt deflation. When more than a few eigenvalues are sought, successive iterations through Arnoldi's algorithm fail to improve or maintain the accuracy of the eigenvalues converged in the previous iterations. Therefore, the converged eigenvalues are eliminated from the subsequent iterations by the Schur-Weilandt deflation process [29]. If $p$ eigenvectors $\boldsymbol{x}_{1}, \boldsymbol{x}_{2}, \ldots, \boldsymbol{x}_{p}$ corresponding to the eigenvalues $c_{1}, c_{2}, \ldots, c_{p}$ of $\boldsymbol{A}$ are known, the deflated matrix is

$$
\boldsymbol{A}_{p}=\boldsymbol{A}-\boldsymbol{X}_{p} \sum_{p} \boldsymbol{X}_{p}^{\mathrm{H}}
$$

where $\boldsymbol{X}_{p}=\left\{\boldsymbol{x}_{1}, \boldsymbol{x}_{2}, \ldots, \boldsymbol{x}_{p}\right\}, \Sigma_{p}=\operatorname{diag}\left\{\boldsymbol{\sigma}_{1}, \boldsymbol{\sigma}_{2}, \ldots, \boldsymbol{\sigma}_{p}\right\}$, and $\boldsymbol{A}_{p}$ has eigenvalues $c_{k}^{\prime}=c_{k}-\sigma_{k}$ for $k \leq p$ and $c_{k}^{\prime}=c_{k}$ for $k>p$. The eigenvectors of $\boldsymbol{A}_{p}$ are identical to those of $\boldsymbol{A}$. Saad did not form the deflated matrix $\boldsymbol{A}_{p}$ explicitly, because it is a full matrix even when $\boldsymbol{A}$ is sparse. Instead, $\boldsymbol{A}_{p}$ is computed in a factored form, in which a new Hessenberg matrix is con- 
structed, such that

$$
\boldsymbol{H}_{p+m}=\left[\boldsymbol{X}_{p}: \boldsymbol{Q}_{m}\right]^{\dagger} \boldsymbol{A}\left[\boldsymbol{X}_{p}: \boldsymbol{Q}_{m}\right]=\left[\begin{array}{ccc}
\boldsymbol{X}_{p}^{\dagger} \boldsymbol{A} \boldsymbol{X}_{p} & \vdots & \boldsymbol{X}_{p}^{\dagger} \boldsymbol{A} \boldsymbol{Q}_{m}^{\dagger} \\
\cdots & \vdots & \cdots \\
\boldsymbol{Q}_{m}^{\dagger} \boldsymbol{A} \boldsymbol{X}_{p} & \vdots & \boldsymbol{Q}_{m}^{\dagger} \boldsymbol{A} \boldsymbol{Q}_{m}
\end{array}\right]
$$

The matrix $A$ can also be represented in an orthogonal subspace spanned by $\boldsymbol{U}=\left\{\boldsymbol{u}_{1}, \boldsymbol{u}_{2}, \ldots, \boldsymbol{u}_{m}\right\}$, such that $\boldsymbol{R}=\boldsymbol{U}^{\dagger} \boldsymbol{A} \boldsymbol{U}$, where $\boldsymbol{R}$ is an $m \times m$ upper quasi-triangular matrix. The subspace $U$ is called Schur subspace following Schur's theorem [32]. When the basic algorithm and the modifications are carried out in terms of Schur vectors, the Hessenberg matrix $\boldsymbol{H}_{p+m}$ can be written as

$$
\boldsymbol{H}_{p+m} \approx\left[\begin{array}{ccc}
\boldsymbol{R}_{p} & \vdots & \boldsymbol{U}_{p}^{\dagger} \boldsymbol{A} \boldsymbol{Q}_{m} \\
\cdots & \vdots & \cdots \\
0 & \vdots & \boldsymbol{H}_{m}
\end{array}\right]
$$

where the diagonal $2 \times 2$ submatrices of the upper quasi-triangular matrix $\boldsymbol{R}_{p}$ provide the real and imaginary parts of the computed $p$ eigenvalues, and $H_{m}$ has the remaining eigenvalues of $\boldsymbol{H}_{p+m}$.

As mentioned earlier, a transformation $\boldsymbol{P}(\boldsymbol{J}, \boldsymbol{M})$ is needed that maps the leading eigenvalues to the outermost part of the eigenspectrum of $\boldsymbol{P}(\boldsymbol{J}, \boldsymbol{M})$. The transformation is chosen here such that eigenvalues $\lambda$ of $\boldsymbol{P}$ are exponentially related to the eigenvalues $c$ of the eigenproblem $\boldsymbol{J x}=c \boldsymbol{M x}$ by $\lambda=$ $\exp (c t)$, where $t$ is a positive real parameter. Such a transformation, called pre-conditioning, clearly maps the leading eigenvalues of $\boldsymbol{J} \boldsymbol{x}=\boldsymbol{c M x}$ to the dominant eigenvalues of $\boldsymbol{P}$. For computational purposes, the exponential transformation is approximated by a polynomial [30] that can be expressed as,

$$
\boldsymbol{P}(\boldsymbol{J}, \boldsymbol{M}, \boldsymbol{\theta}, t, k)=\left[\boldsymbol{M}-\frac{\boldsymbol{J}(1-\boldsymbol{\theta}) t}{k}\right]^{-k}\left[\boldsymbol{M}+\frac{\boldsymbol{J} \theta t}{k}\right]^{k} .
$$

The transformation is analogous to a finite-difference integration of a transient problem $\boldsymbol{M} \dot{\boldsymbol{w}}=\boldsymbol{J} \boldsymbol{w}$ using the $\boldsymbol{\theta}$-method. The solution after $k$ time steps would be represented as $\boldsymbol{w}(t)=\boldsymbol{P}(\boldsymbol{J}, \boldsymbol{M}, \boldsymbol{\theta}, t, k) \boldsymbol{q}_{1}$, where $\boldsymbol{w}(t=0)=$ $q_{1}$. The analogy is useful in providing suitable values for the parameters $t, \theta$, and $k$. When $\Delta t=t / k$ is large, the convergence is accelerated, but not necessarily to the leading eigenmodes. When $\Delta t$ is small, the exponentials of the eigenvalues are computed accurately, and the leading modes are projected out. However, for small $\Delta t$, the eigenvalues of $\boldsymbol{P}$ tend to cluster near unity, and more steps $k$ are, therefore, required to separate them, which increases the computational cost. 
In the interfacial modes of instability in multilayer extrusion, the growth factor is smaller by four to five orders of magnitude than the oscillation speed $[6,7]$. Therefore, $\Delta t$ needs to be smaller by at least four to five orders of magnitude compared to the time required to observe any significant growth in disturbances. In other words, small $\Delta t$ and large $k$ are needed for accurate mapping of the leading modes to the dominant modes. The above stiff problem is resolved by the following two additional modifications.

(1) Acceleration. Instead of using an arbitrary initial unit vector $\boldsymbol{q}_{1}$, integration is performed before passing the new $\boldsymbol{q}_{1}^{k}$ to Arnoldi's algorithm, where

$\boldsymbol{q}_{1}^{k}=\boldsymbol{P}(\boldsymbol{J}, \boldsymbol{M}, \boldsymbol{\theta}=0, \Delta t, k) \boldsymbol{q}_{1}$.

The acceleration filters out very large artificial eigenvalues arising due to the singular $\boldsymbol{M}$, and also projects out leading components of the initial vector [33].

(2) Complex shift. To separate the leading eigenvalues of a stiff problem that cluster near unity in the exponential transformation, the eigenproblem is shifted such that,

$\boldsymbol{P}(\boldsymbol{J}, \boldsymbol{M}, \boldsymbol{\sigma}, \boldsymbol{\theta}, \Delta t, k)=[\boldsymbol{M}-\boldsymbol{B}(1-\theta) \Delta t]^{-k}[\boldsymbol{M}+\boldsymbol{B} \boldsymbol{\theta} \Delta t]^{k}$,

where $\boldsymbol{B}=(\boldsymbol{J}-\boldsymbol{\sigma} \boldsymbol{M})$, and $\sigma$ is the complex shift. The transformation favors the leading eigenvalues that are closest to $\sigma$. Therefore, when the computations are done by changing parameters marginally, $\sigma$ can be chosen to be the leading eigenvalue of the previous calculations.

In the acceleration or in the pre-conditioning, $\boldsymbol{q}_{\text {new }}=\boldsymbol{q}_{k+1}$, should be computed by solving (refer to eqn. (25)),

$$
[\boldsymbol{M}-\boldsymbol{B}(1-\boldsymbol{\theta}) \Delta t] \boldsymbol{q}^{l+1}=[\boldsymbol{M}+\boldsymbol{B} \boldsymbol{\theta} \Delta t] \boldsymbol{q}^{l}, \quad l=1,2, \ldots, k,
$$

sequentially. Equation (27) can also be written as

$$
[\boldsymbol{M}-\boldsymbol{B}(1-\theta) \Delta t]\left(\boldsymbol{q}^{l+1}+a \boldsymbol{q}^{l}\right)=(a+1) \boldsymbol{M} \boldsymbol{q}^{l},
$$

where $a=\theta /(1-\theta)$. The matrix-vector product $\boldsymbol{M q ^ { l }}$ is approximately equal to the residual vector $-\boldsymbol{R}\left(\boldsymbol{x}=\boldsymbol{x}_{0}, \dot{\boldsymbol{x}}=\boldsymbol{q}^{l}\right)$ [30]. Thus, an expensive matrix-vector multiplication can be avoided by evaluating the residual vector $\boldsymbol{R}\left(\boldsymbol{x}=\boldsymbol{x}_{0}, \dot{\boldsymbol{x}}=\boldsymbol{q}^{\prime}\right)$. The LU decomposition of $[\boldsymbol{M}-\boldsymbol{B}(1-\theta) \Delta t]$ is carried out once, and at each subsequent step, the right-hand side of eqn. (28) is evaluated and backward substitution is performed. The sparseness is maintained, and the frontal solver can be elegantly incorporated $[19,20]$.

Once the most dominant eigenvalues of the transformed problem are calculated by Arnoldi's algorithm, the leading eigenvalues of the original problem are then estimated by using the fact that the eigenvectors are identical in both the problems. A less expensive, though probably less 
accurate way of evaluating the eigenvalues is to pick only one component of $\boldsymbol{J} \boldsymbol{x}=\boldsymbol{c M x}$, containing non-zero time-derivatives, to calculate $c$. Then, for real $\boldsymbol{x}$,

$\sum_{j} J_{k l} x_{l}=c \sum_{l} M_{k l} x_{l}$

is solved, and for complex $\boldsymbol{x}$,

$\sum_{j} J_{k l} x_{\mathrm{R} l}=c_{\mathrm{R}} \sum_{l} M_{k l} x_{\mathrm{R} l}-c_{\mathrm{I}} \sum_{l} M_{k l} x_{\mathrm{I} l}$,

and

$\sum_{j} J_{k l} x_{\mathrm{I} l}=c_{\mathrm{R}} \sum_{l} M_{k l} x_{\mathrm{I} l}+c_{\mathrm{I}} \sum_{l} M_{k l} x_{\mathrm{R} l}$

are solved simultaneously.

In summary, the steps to be followed for the calculations of the leading eigenvalues are:

(1) selection of various parameters required in the algorithm;

(2) acceleration;

(3) iterative Arnoldi's algorithm with pre-conditioning, deflation, complex shift and reorthogonalization;

(4) computation of leading eigenvalues using eqn. (29).

If the computed eigenvalues do not appear in a sequence of decreasing real parts and increasing residual norms, $\Delta t$ is decreased, $k$ is increased, more steps are used in acceleration and in pre-conditioning, and the whole process is repeated.

\section{Results}

The steady-state variables are first calculated at certain operating and rheological parameters, and then the leading eigenvalues are estimated to determine the stability of the flow. The analysis is carried out at several different conditions by gradually changing the parameters. First-order continuation is used to predict the new initial estimates, and the previously estimated leading eigenvalues are used as the complex shift. All the calculations are performed on an IBM3090/600E mainframe computer, either at the University of Michigan, or at the Cornell Supercomputer Center.

The ratios of steady-state thicknesses of individual layers remain the same across the length of the converging die at all operating conditions. This result is as expected for long dies with small variations in the total depth along the length, and for the flows at small Reynolds numbers $\left(10^{-6} \leq R e_{j}\right.$ $\leq 0.5$ ) encountered in coextrusion. Thus, the results of the steady-state analysis are trivial, and will not be presented here. 
The dimension $m$ of Krylov subspaces in Arnoldi's algorithm was set to either 30 or 60 depending on the accuracy of the initial estimates of the leading eigenspectrum, and on the number $r$ of the leading eigenmodes sought in the analysis. On most occasions, it was sufficient to compute the first 18 leading modes. On a few occasions, however, the computed modes did not reveal all the first 18 leading modes. In those cases, it was necessary to increase the number of wanted modes to 30 or 35 with a suitable increase in $m$. The implicitness factor $\theta$ was always set to zero in all the cases for both preconditioning and acceleration. The imaginary part of the leading eigenvalues is larger by four orders of magnitude compared to the real part. Therefore, the number of steps $k$ in both pre-conditioning and acceleration was at least equal to 25 . The complex shift parameter $\sigma$ was set to $\bar{\lambda}_{\text {lead }}$, the best current approximation to the leading eigenvalue. The only criterion used in determining a suitable value of $\Delta t$ was a possibility of the existence of any leading mode outside the computed leading eigenspectrum.

A refinement in the tessellation of the finite element flow domain is a very crucial factor in the linear stability analysis. The discretization gives rise to spurious eigenvalues that are not close to any eigenvalues of the continuous problem [18]. These eigenvalues are very sensitive to the tessellation, and disappear with refinement. In addition, for $L \gg H$, the amplitude of the disturbances for a given eigenmode is spatially approximately periodic along the direction of flow. Therefore, the eigenvalues associated with the disturbances of very small periods (or of large wavenumbers) are suppressed in coarser discretizations, even though they are the leading ones. As a finer and finer tessellation is used, these eigenvalues start appearing in the leading eigenspectrum. Therefore, if the leading eigenvalues are associated with high wavenumbers for a given set of parameters, a very fine tessellation is necessary.

Although the complex shift was implemented, real arithmetic was used in the algorithm for real $\boldsymbol{M}$ and $\boldsymbol{J}$. Therefore, the computational cost should be no more than twice the cost of calculations using the real shift. However, there are several other factors that increase the computational cost enormously. These factors are:

(1) the large values of the numerical parameters, such as the dimension $m$ of Krylov subspace, the total number $r$ of desired leading eigenvalues, and the parameter $k$, compared to a similar analysis of coating flows by Christodoulou and Scriven [30],

(2) the large number of elements due to a finer tessellation, and due to multiple flow domains in our analysis;

(3) the large number of unknowns in the analysis of viscoelastic flows, where the components of stress tensor are also unknowns along with velocity vector, pressure and streamline locations. 


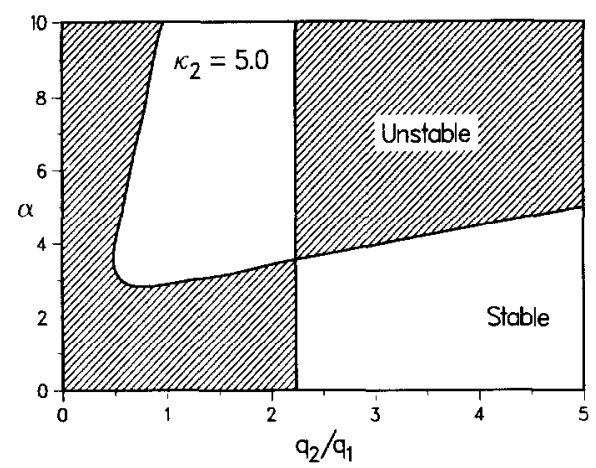

Fig. 4. Neutral stability curves for two-layer flow in the $\alpha-q_{2} / q_{1}$ plane for $\kappa_{2}=5.0$ by the 1-D approach. The other parameters are $R e_{1}=5.0, C a_{1}=\infty$ and $S t=0.0$. The hatched regions are unstable and the unhatched regions are stable.

Therefore, the number of layers in coextrusion is restricted to two, and the analysis is carried out for a very limited number of cases in flows with only two layers.

Before any computations are done for the flow in converging channels, the results of the 2-D and 1-D approaches are compared for two-layer flow of Newtonian and viscoelastic liquids in channels of constant depth to check the validity of Arnoldi's algorithm (see Table 1 for the comparison between these two approaches). The die is ten times longer than the depth, and the liquids in all layers are assumed to have the same density in all further calculations. For the Newtonian flow, the parameters are $\kappa_{2}=5.0, R e_{1}=5.0$, $S t=0.0$, and $C a_{1}=\infty$. The neutral stability diagram of this flow at various wavenumbers by the 1-D approach [4] is shown in Fig. 4, whereas the details of the discretization in the 2-D approach are tabulated in Table 2. The converged leading eigenspectrum changed with refinement, and a mesh-in-

\section{TABLE 2}

Discretization of the flow domain in the linear stability analysis of two-layer Newtonian flow by the 2-D approach

\begin{tabular}{llcc}
\hline Number of elements & & Total & $\begin{array}{l}\text { Number of } \\
\text { unknowns }\end{array}$ \\
\hline Along $x$ & $\begin{array}{l}\text { Along } y \text { in } \\
\text { each layer }\end{array}$ & & 781 \\
\hline 10 & 3 & 60 & 1521 \\
20 & 3 & 120 & 3893 \\
40 & 4 & 320 & 7145 \\
60 & 5 & 600 & \\
\hline
\end{tabular}




\section{TABLE 3}

Discretization of the flow domain in the linear stability analysis of two-layer viscoelastic flows by the 2-D approach

\begin{tabular}{|c|c|c|c|c|}
\hline \multicolumn{3}{|c|}{ Number of elements } & \multirow{2}{*}{$\begin{array}{l}\text { Number of } \\
\text { unknowns }\end{array}$} & \multirow{2}{*}{$\begin{array}{l}\text { CPU time } \\
\text { (s) }\end{array}$} \\
\hline Along $x$ & $\begin{array}{l}\text { Along } y \text { in } \\
\text { in each layer }\end{array}$ & Total & & \\
\hline 10 & 3 & 60 & 1663 & 15 \\
\hline 20 & 3 & 120 & 3243 & 40 \\
\hline 40 & 4 & 320 & 8587 & 180 \\
\hline
\end{tabular}

dependent leading eigensolution was never found for any $\boldsymbol{q}_{2} / \boldsymbol{q}_{1}$. From Fig. 4 , leading (stable or unstable) eigenvalues always exist at very large wavenumbers in Newtonian flows, which start appearing in the leading eigenspectrum of the refined domain. Therefore, a mesh-independent leading eigenspectrum cannot be computed. Although the lack of a mesh-independent eigensolution indicates the existence of a leading eigensolution at large wavenumbers, it does not necessarily indicate the lack of stability.

The details of the successive refinement and the approximate CPU time required for one iteration for two-layer flow of viscoelastic liquids by the 2-D approach is listed in Table 3. The parameters are $\kappa_{2}=5.0, R e_{1}=0.1$, $S t=0.0, C a_{1}=\infty, \Lambda_{11}=0.001, \Lambda_{12} / \Lambda_{11}=0.1, \Lambda_{v 1}=\Lambda_{v 2}=1.0, n_{1}=n_{2}$, and the values of the power-law exponents are in the range 0.9 to 0.3 . No new leading eigenvalues appeared when the mesh was refined from 120 elements to 320 elements, and all the spurious eigenvalues are suppressed. Thus, refinement up to 120 elements is sufficient for these calculations. Note that the total CPU time depends on the order of the system, the numerical parameters $r, m$ and $k$, and the number of iterations (which depends on the accuracy of the initial estimate). However, once the LU decomposition is performed, subsequent computations can be carried out by only modifying the right-hand side vector. The order of the system and the values of the numerical parameters are very large. Therefore, the total computational cost of analyzing the stability of this stiff problem is excessive.

The neutral stability diagrams of the viscoelastic flow under considerations are shown in Fig. 5 using the 1-D approach [6,7]. The critical flow-rate ratios $\left(q_{2} / q_{1}\right)_{\text {critl }}$ in these diagrams are the flow rates beyond which the flow is stable at all wavenumbers. Similar critical flow-rate ratios are also computed using the 2-D approach. When $q_{2} / q_{1}$ was gradually decreased from a very high value to a very low value, the real part of the leading eigenvalue crosses the imaginary axis, and becomes positive at the critical flow-rate ratio $\left(q_{2} / q_{1}\right)_{\text {crit2 }}$. These critical flow-rate ratios $\left(q_{2} / q_{1}\right)_{\text {crit }}$ and $\left(q_{2} / q_{1}\right)_{\text {crit2 }}$ are compared in Table 4 . The imaginary part of the leading 

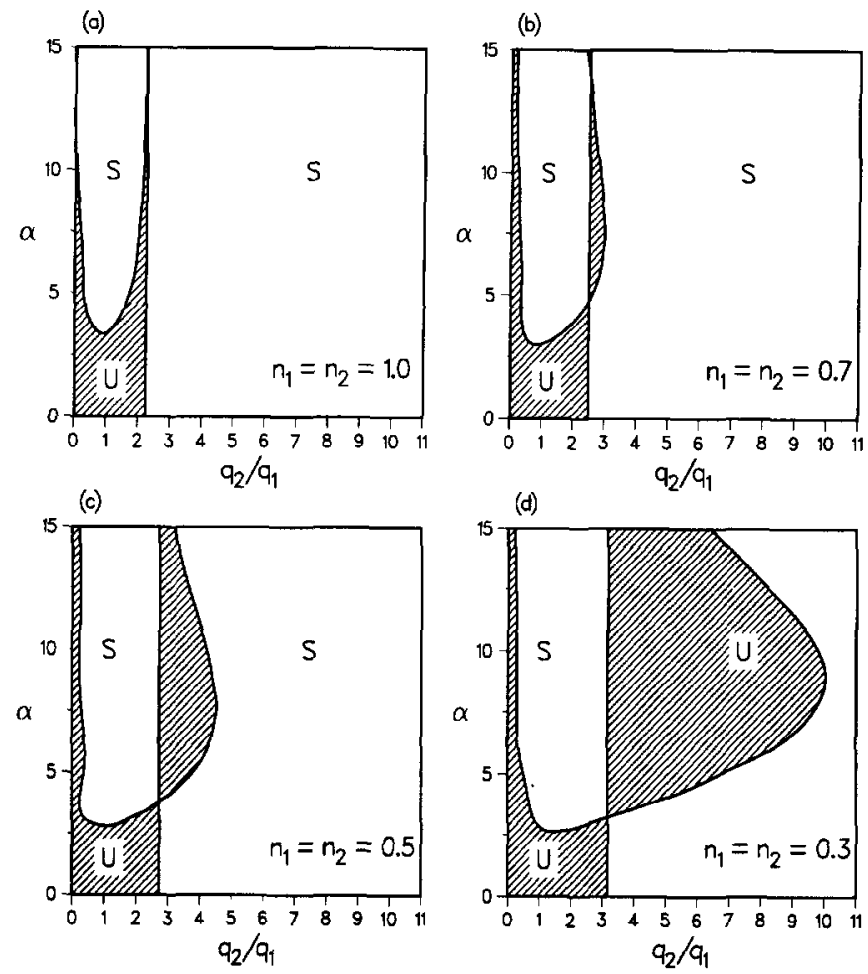

Fig. 5. Neutral stability curve for two-layer flow at various power-law exponents $n_{1}=n_{2}$. The parameters are $R e_{1}=0.1, \kappa_{2}=5.0, \Lambda_{11}=0.001, \Lambda_{12} / \Lambda_{11}=0.1$, and $\Lambda_{v 1}=\Lambda_{v 2}=1.0$. Hatched regions are unstable and unhatched regions are stable.

\section{TABLE 4}

Comparison between the critical flow-rate ratios $\left(q_{2} / q_{1}\right)_{\text {crit }}$ estimated by the 2-D approach, and $\left(q_{2} / q_{1}\right)_{\text {critl }}$ computed by the 1-D approach at various power-law exponents $n_{j}$ for two-layer flow in a channel of constant depth

\begin{tabular}{llcrc}
\hline$n_{1}=n_{2}$ & $\left(q_{2} / q_{1}\right)_{\text {crit }}$ & $\left(q_{2} / q_{1}\right)_{\text {crit }}$ & $c_{\mathrm{I}, \text { crit } 2}$ & $c_{\mathrm{I}, \mathrm{crit} 1}$ \\
\hline 1.0 & - & 2.10 & - & - \\
0.7 & 2.91 & 2.97 & 10.10 & 10.54 \\
0.5 & 4.10 & 4.48 & 8.91 & 9.84 \\
0.3 & 8.80 & 10.05 & 7.23 & 9.70 \\
\hline
\end{tabular}

The imaginary parts of the leading eigenvalues at the critical flow-rate ratios by these two approaches are also compared. The other parameters are $\kappa_{2}=5.0, \operatorname{Re}_{1}=0.1, S t=0.0$, $C a_{1}=\infty, \Lambda_{11}=0.001, \Lambda_{12} / \Lambda_{11}=0.1, \Lambda_{v 1}=\Lambda_{v 2}=1.0$, and $n_{1}=n_{2}$. 


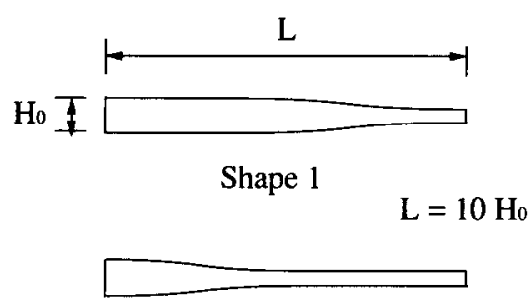

Shape 2

Fig. 6. Actual shapes of the converging channels drawn to the scale.

eigenvalue $c_{\mathrm{I}, \mathrm{cr} t 2}$ at the point where the real part crosses the zero line, is also compared in Table 4 with its counterpart $c_{\mathrm{I}, \text { critl }}$ in the 1-D approach. The critical flow-rate ratio $\left(q_{2} / q_{1}\right)_{\text {crit } 2}$ and the imaginary part of the leading eigenvalue $c_{\text {I,crit2 }}$ compare well with $\left(q_{2} / q_{1}\right)_{\text {crit1 }}$ and $c_{\text {I,crit1 }}$ respectively, at all values of $n_{1}$ and $n_{2}$, except at $n_{1}=n_{2}=1.0$, where $\left(q_{2} / q_{1}\right)_{\text {crit } 2}$ could not be estimated. As $n_{1}$ and $n_{2}$ decrease, the accuracy of Arnoldi's algorithm decreases. However, the difference between $\left(q_{2} / q_{1}\right)_{\text {crit2 }}$ and $\left(q_{2} / q_{1}\right)_{\text {crit1 }}$ is always less than $15 \%$. At $n_{1}=n_{2}=1.0,\left(q_{2} / q_{1}\right)_{\text {crit1 }}$ is determined by a straight line of constant $q_{2} / q_{1}$ (see Fig. 5(a)). In other words, there is a large number of leading eigenvalues with a wide range of imaginary parts that can become unstable at $\left(q_{2} / q_{1}\right)_{\text {crit1 }}$. Therefore, several leading eigenvalues cluster together in the spectral transformation, which could not be resolved even by using extremely high values of $r, m$, and $k$. In conclusion, the results obtained by using Arnoldi's algorithm (2-D) compare well with the results obtained by the pseudospectral method (1-D) for two-layer channel flow of viscoelastic liquids except at $n_{1}=n_{2}=1.0$. The detailed discussion on the interpretation of the effects of the rheological and operating parameters on the stability of the flow is beyond the scope of this work, and can be found in refs. 4, 6 and 7.

Two shapes of converging channels (see Fig. 6 for the diagrams of the converging channels drawn to scale) are investigated for two values of the ratio $H_{\text {out }} / H_{0}$ of depth at the outlet to that at the inlet of the die. The die is ten times longer than the depth at the inlet of the die. The calculations discussed above are repeated for these flow domains. The numerical parameters and discretization are the same as those in earlier calculations of viscoelastic flows. The parameters are $\kappa_{2}=5.0, R e_{1}=0.1, S t=0.0, C a_{1}=\infty$, $\Lambda_{11}=0.001, \Lambda_{12} / \Lambda_{11}=0.1, \Lambda_{v 1}=\Lambda_{v 2}=1.0$, and $n_{1}=n_{2}$. The critical flow-rate ratio for both shapes of the converging channels and for two values of $H_{\text {out }} / H_{0}$ at various $n_{1}=n_{2}$ are tabulated in Table 5 .

When $\left(q_{2} / q_{1}\right)_{\text {crit2 }}$ of the channel flow $\left(H_{\text {out }} / H_{0}=1.0\right)$ are compared to those of the flow through converging channels $\left(H_{\text {out }} / H_{0} \neq 1.0\right)$, no conclusive variations in $\left(q_{2} / q_{1}\right)_{\text {crit2 }}$ with respect to the shapes of the die, or with 


\section{TABLE 5}

Critical flow-rate ratios for various values of power-law exponents $n_{l}$, and for various depth ratios at the outlet to the inlet of the die in two-layer flow of viscoelastic liquids

\begin{tabular}{llcc}
\hline$n_{1}, n_{2}$ & $H_{\text {out }} / H_{0}$ & $\left(q_{2} / q_{1}\right)_{\text {crit } 2}$ & \\
\cline { 3 - 4 } & & Shape 1 & Shape 2 \\
\hline 0.7 & 1.00 & 2.91 & \\
& 0.75 & 2.88 & 2.80 \\
& 0.50 & 2.93 & 2.96 \\
0.5 & 1.00 & 4.10 & 4.20 \\
& 0.75 & 4.28 & 4.22 \\
& 0.50 & 4.16 & \\
0.3 & 1.00 & 8.80 & 9.60 \\
& 0.75 & 8.20 & 11.20 \\
\hline
\end{tabular}

The parameters are $\kappa_{2}=5.0, R e_{1}=0.1, S t=0.0, C a_{1}=\infty, \Lambda_{11}=0.001, \Lambda_{12} / \Lambda_{11}=0.1$, $\Lambda_{\iota 1}=\Lambda_{v 2}=1.0$, and $n_{1}=n_{2}$.

(c)

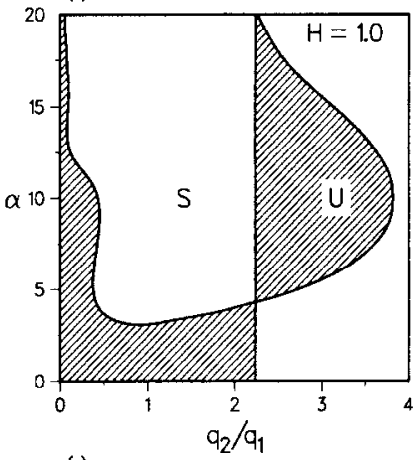

(c)

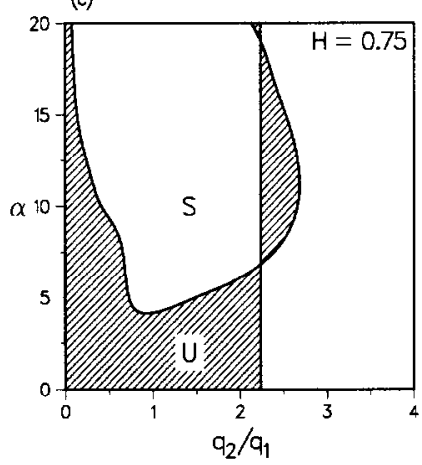

(b)
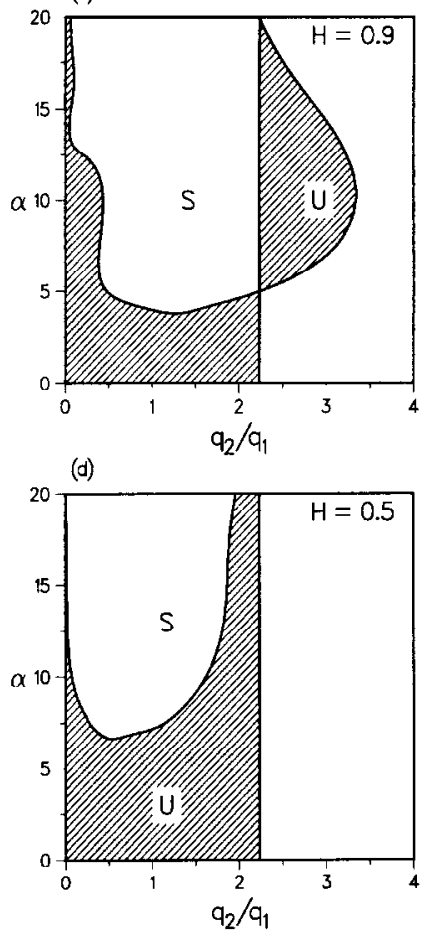

Fig. 7. Neutral stability curves for two-layer flow at various sections in the converging channel (at various depths $H$, nondimensionalized with depth $H_{0}$ at the inlet) for $n_{1}=n_{2}=1.0$. The parameters are $R e_{1}=0.1, \kappa_{2}=5.0, \Lambda_{11}=0.001, \Lambda_{12} / \Lambda_{11}=0.1$, and $\Lambda_{v 1}=\Lambda_{v 2}=1.0$. The hatched regions are unstable and the unhatched regions are stable. 

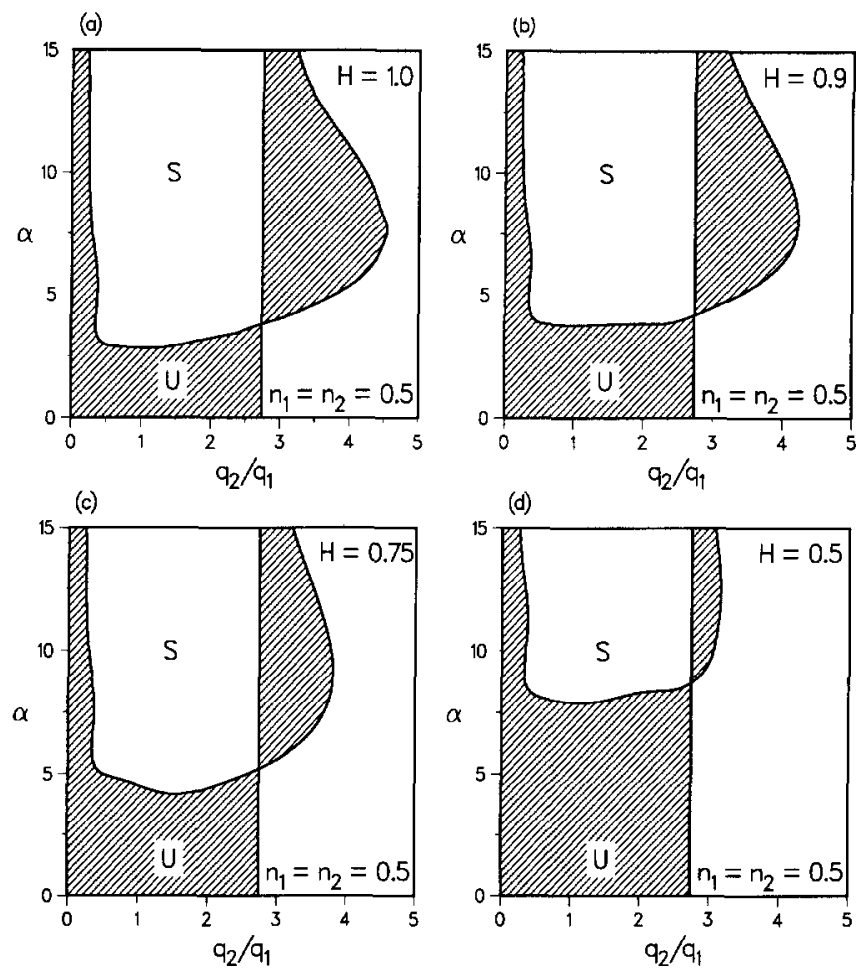

Fig. 8. Neutral stability curves for two-layer flow at various sections in the converging channel (at various depths $H$, nondimensionalized with depth $H_{\text {in }}$ at the inlet) for $n_{1}=n_{2}=$ 0.5. The parameters are $R e_{1}=0.1, \kappa_{2}=5.0, \Lambda_{11}=0.001, \Lambda_{12} / \Lambda_{11}=0.1$, and $\Lambda_{v 1}=\Lambda_{v 2}=1.0$. The hatched regions are unstable and the unhatched regions are stable.

respect to $H_{\text {out }} / H_{0}$ are observed in Table 5, indicating that the leading eigenvalues are associated with the depth of the die at the inlet. Although no particular trend in $\left(q_{2} / q_{1}\right)_{\text {crit2 }}$ is observed at $n_{1}=n_{2}=0.3$, the values of $\left(q_{2} / q_{1}\right)_{\text {crit2 }}$ differ for the two shapes and for two values of $H_{\text {out }} / H_{0}$. We believe that the difference is due to the inaccuracy involved in computing the unknowns for shear-thinning liquids, which is observed in other flows as well [34].

Since the die is longer by an order of magnitude than its depth, the 1-D approach can also be used to find the critical flow-rate ratio $\left(q_{2} / q_{1}\right)_{\text {critl }}$. The results in terms of the neutral stability diagrams for various sections are shown in Figs. 7 and 8. The power-law exponents $n_{1}$ and $n_{2}$ are equal to 1.0 in Fig. 7, and are equal to 0.5 in Fig. 8. The other parameters are $\kappa_{2}=5.0$, $R e_{1}=0.1, S t=0.0, C a_{1}=\infty, \Lambda_{11}=0.001, \Lambda_{12} / \Lambda_{11}=0.1, \Lambda_{v 1}=\Lambda_{v 2}=1.0$, and $n_{1}=n_{2}$. The critical flow-rate ratio in both the figures is the highest for the section with the maximum depth $(H=1.0)$, which corresponds to the 
inlet of the die. Thus, both 2-D and 1-D approaches provide identical critical flow-rate ratios, which correspond to the maximum depth of the channel. Thus, computationally expensive 2-D analysis is not needed for multilayer extrusion through long, converging dies. However, such conclusions can be arrived at only after comparing the results from the 2-D and 1-D approaches. When the depth of the channel is decreased, the Reynolds number does not change. However, the dimensionless relaxation time decreases. Since the relaxation time has a stabilizing effect on the stability of multilayer extrusion [6,7], the maximum destabilizing effect is observed at the maximum depth of the channel. Besides, as suggested by Khomami [8], normal stresses generated by the converging channel provide a stabilizing effect in multilayer flows.

\section{Conclusions}

A two-dimensional linear stability analysis of two-dimensional multilayer flow of viscoelastic liquids through long, converging channels is conducted (2-D). A modified Oldroyd-B model with shear-rate dependent viscosity, represented by the Carreau viscosity function, is used as the constitutive equation. Since the length of the channel is an order of magnitude larger than its depth, a simplified analysis, in which one-dimensional channel flow is assumed locally at various cross-sections of the die, is also performed (1-D).

The resulting generalized eigenvalue problem in the 2-D approach is sparse, banded, large (of the order of 3000-8000), and has a singular mass matrix. The difficult task of computing the leading eigenvalues of such a generalized eigenvalue problem is carried out using a sophisticated iterative Arnoldi's algorithm. Schur-Weilandt deflation, complex shift, acceleration and exponential pre-conditioning are incorporated to map the leading eigenvalues to the outer spectrum of the transformed problem, and to make the algorithm sufficiently flexible to solve the stiff problem of interfacial instability in multilayer extrusion, thereby establishing the applicability of the algorithm for any large hydrodynamic stability problem.

The critical flow-rate ratios are estimated using Arnoldi's algorithm for two shapes of the converging die at two different ratios $\left(H_{\text {out }} / H_{0}\right)$ of depths at the outlet to the inlet of the die. The critical flow-rate ratios are observed to be independent of either the shapes or the ratios $\left(H_{\text {out }} / H_{0}\right)$. Thus, both 2-D and 1-D approaches provide identical critical parameters, which are associated with the inlet of the die. Thus, the most important advantage of comparing the results obtained from both the approaches is that the critical parameters of interfacial instability in multilayer extrusion through long, converging dies can be determined by a simplified and computationally 
inexpensive 1-D approach. However, a priori validation of the results from the 1-D approach is necessary by means of the 2-D approach described in this paper.

\section{Acknowledgements}

This research was funded by a grant from the Dow Chemical Company, Midland, Michigan, whose support is gratefully acknowledged. We also wish to acknowledge valuable discussions with Dr. Christodoulou and Professor Saad.

\section{References}

1 C.S. Yih, Instability due to Viscosity Stratification, J. Fluid Mech., 27 (1967) 337.

2 S.G. Yiantsios and B.G. Higgins, Linear Stability of Plane Poiseuille Flow of Two Superposed Fluids: Shear and Interfacial Modes, Phys. Fluids, 31 (1988) 3225.

3 A.P. Hooper, The stability of Two Superposed Fluids in a Channel, Phys. Fluids, Part A, 1 (1989) 1133.

4 N.R. Anturkar, T.C. Papanastasiou and J.O. Wilkes, Linear Stability Analysis of Multilayer Plane Poiseuille Flow, Phys. Fluids, Part A, 2 (1990) 530.

5 N.D. Waters and A.M. Keeley, The Stability of Two Stratified Non-Newtonian Liquids in Couette Flow, J. Non-Newtonian Fluid Mech., 24 (1987) 161.

6 N.R. Anturkar, T.C. Papanastasiou and J.O. Wilkes, Stability of Multilayer Extrusion of Viscoelastic Liquids, AIChE J., 36 (1990) 710.

7 N.R. Anturkar, T.C. Papanastasiou and J.O. Wilkes, Errata, AIChE J., 36 (1990) 1609.

8 B. Khomami, Interfacial Stability and Deformation of Two Stratified Power Law Fluids in Plane Poiseuille Flow. Part II. Interface Deformation, J. Non-Newtonian Fluid Mech., 37 (1990) 19.

9 C.D. Han and R. Shetty, Studies of Multilayer Film Coextrusion. II. Interfacial Instability in Flat Film Coextrusion, Polym. Eng. Sci., 18 (1978) 180.

10 T.W. Kao and C. Park, Experimental Investigations of the Stability of Channel Flows. 2: Two layered Concurrent Flow in a Rectangular Channel, J. Fluid Mech., 52 (1972) 401.

11 R. Ramanathan, personal communication, The Dow Chemical Company, Midland, Michigan, 1990.

12 R.B. Bird, R.C. Armstrong and O. Hassager, Dynamics of Polymeric Liquids, Vol. 1, Fluid Mechanics, 2nd edn., Wiley, New York, 1987.

13 M.J. Crochet, and R. Keunings, Finite Element Analysis of Die Swell of a Highly Elastic Fluid, J. Non-Newtonian Fluid Mech., 10 (1982) 339.

14 R.W. Wesson and T.C. Papanastasiou, Flow Singularity and Slip Velocity in Plane Extrudate Swell Computations, J. Non-Newtonian Fluid Mech., 26 (1988) 277.

15 T.C. Papanastasiou, C.W. Macosko and L.E. Scriven, Analysis of Lubricated Squeezing Flows, Int. J. Numer. Methods Fluids, 6 (1986) 819.

16 N. Malamataris, K.R.J. Ellwood and T.C. Papanastasiou, A New Outflow Boundary Condition, Int. J. Numer. Methods Fluids, in press.

17 T.C. Papanastasiou, C.W. Macosko and L.E. Scriven, Streamlined finite elements and transit times, in R.H. Gallagher, G.F. Carey, J.T. Oden, and O.C. Zienkiewicz (Eds), Finite Elements in Fluids, Vol. 6, Wiley, New York, 1985, p. 257. 
18 G. Strang and G.J. Fix, An Analysis of the Finite Element Method, Prentice-Hall, Englewood Cliffs, NJ, 1973.

19 P. Hood, Frontal Solution Program for Unsymmetric Matrices, Int. J. Num. Methods Eng., 10 (1976) 379.

20 P. Hood, Correction, Int. J. Numer. Methods Eng., 11 (1977) 1055.

21 P.G. Drazin and W.H. Reid, Hydrodynamic Stability, Cambridge University Press, Cambridge, 1981.

22 N.E. Bixler, Stability of a Coating Flow, Ph.D. Thesis, University of Minnesota, Minneapolis, 1982.

23 J.G.F. Francis, The QR Transformation, Part I, Comput. J., 4 (1961) 265.

24 J.G.F. Francis, The QR Transformation, Part II, Comput. J., 4 (1962) 322.

25 G.W. Stewart, Introduction to Matrix Computations, Academic Press, New York, 1973.

26 C. Lanczos, An Iteration Method for the Solution of the Eigenvalue Problem of Linear, Differential and Integral Operator, J. Res. Nat. Bur. Stand., 45 (1950) 255.

27 W.E. Arnoldi, The Principle of Minimized Iteration in the Solution of the Matrix Eigenvalue Problem, Q. Appl. Math., 9 (1951) 17.

28 G.H. Golub and C.F. Van Loan, Matrix Computations, Johns Hopkins University Press, Baltimore, 1983.

29 Y. Saad, Numerical solution of large unsymmetric eigenvalue problems, in Proc. Workshop on Practical Iterative Methods for Large Scale Computations, University of Minnesota Supercomputer Institute, Minneapolis, Elsevier, Amsterdam, 1988.

30 K.N. Christodoulou and L.E. Scriven, Finding Leading Modes of a Viscous Free Surface Flow: An Asymmetric Generalized Eigenproblem, J. Sci. Comput., 3 (1988) 355.

31 Y. Saad, Variations on Arnoldi's Method for Computing Eigenelements of Large Unsymmetric Matrices, Lin. Alg. Appl., 34 (1980) 269.

32 G.W. Stewart, Simultaneous Iteration for Computing Invariant Subspaces of Non-Hermitian Matrices, Numer. Math., 25 (1976) 123.

33 I. Goldhirsch, S.A. Orszag and B.K. Maulik, An Efficient Method for Computing Leading Eigenvalues of Large Asymmetric Matrices, J. Sci. Comput., 2 (1987) 33.

34 N.R. Anturkar and A. Co, Draw Resonance in Film Casting of Viscoelastic Fluids: A Linear Stability Analysis, J. Non-Newtonian Fluid Mech., 28 (1988) 287. 